\title{
Reflexión sobre la metodología de investigación en el proyecto: argumentación intercultural
}

Beatriz H. Amador Lesmes

\begin{abstract}
Resumen
En este artículo se presenta una reflexión sobre la metodología utilizada en el proyecto de investigación Argumentación intercultural a través del uso del software Dígalo, que se desarrolló en el Colegio Sagrado Corazón de Jesús en Cumaribo (Vichada) en el año 2010. Para esta reflexión se propone la necesidad de replantear las metodologías de la investigación, las técnicas de recolección de información y las estrategias didácticas tradicionales en contextos de bilingüismo e interculturalidad, e introducir metodologías que permitan la construcción de sentido con las comunidades mismas. De este modo, se analizan percepciones sobre los modos de interacción y comunicación de los miembros de la comunidad educativa, se presentan sus características comunicacionales e interculturales en un contexto bilingüe y se plantean elementos teóricos que sustentan las posibilidades de una argumentación intercultural a partir de la relación entre interculturalidad, bilingüismo y la generación de espacios para la argumentación, la discusión, la negociación y el debate. Con lo anterior, se identifica en qué medida a través del uso del software mencionado se puede desarrollar la argumentación intercultural y los efectos de las brechas digitales en la generación del aprendizaje a través de las Tecnologías de la Información y la Comunicación, TIC.
\end{abstract}

Palabras clave: argumentación, interculturalidad, multiculturalidad, bilingüismo, software educativo.

1 Licenciada en Filosofía y Letras y Especialista en docencia universitaria de la Universidad de Caldas. Candidata a Magister en Estudios Culturales de la Pontificia Universidad Javeriana. Tutora y Coordinadora de la Licenciatura en Filosofía de la Universidad Nacional Abierta y a Distancia, UNAD, Colombia. Correo electrónico: beatriz. amador.I@gmail.com, beatriz. amador@unad.edu.co. 


\begin{abstract}
This article presents a reflection on the methodology used in the project of research Intercultural argumentation through the use of software Digalo. It discusses the need to rethink the methodologies of research, data collection techniques and traditional teaching strategies of bilingual and intercultural contexts, such as the educational community of "Sagrado Corazón de Jesús" in Cumaribo (Vichada). Thus, we analyze perceptions of the modes of interaction and communication of the members of the community, which is characterized by the intercultural communication in a bilingual context and raises theoretical elements underpinning possibilities of an intercultural argumentation from the relation between multiculturalism, bilingualism and the creation of stages for argumentation, discussion, negotiation and debate. Consequently, it is identified the extent to which the intercultural argumentation can be achieved by using the mentioned software for the purpose to identify the effects from the digital opening in the generation of learning through the Information and Communication Technology, ICT.
\end{abstract}

Keywords: Argumentation, intercultural, multicultural, educational software.

Recibido: 10 de septiembre de 2010 Aceptado: 23 de octubre de 2010

\title{
Introducción
}

Este artículo surge como reflexión de la metodología diseñada para el desarrollo del proyecto de investigación: Argumentación intercultural a través del uso del software Dígalo, financiado por el Sistema de Investigaciones de la Universidad Nacional Abierta y a Distancia, SIUNAD, en la convocatoria de 2009 y desarrollado en 2010 por el grupo de investigación Cibercultura y Territorio, de la misma universidad. Para el desarrollo de la metodología del proyecto se trabajó con los estudiantes de grado décimo del Colegio Sagrado Corazón de Jesús de Cumaribo (Vichada). El objetivo del proyecto estuvo centrado en la identificación de las características de la argumentación intercultural y en analizar cómo se reflejaba este ejercicio a partir del uso del software Dígalo. ${ }^{2}$ Desde el inicio del proyecto se buscó una definición de argumentación intercultural que pudiera

\footnotetext{
2 Dígalo es un software que permite la argumentación gráfica de argumentos. En él la argumentación está basada en el modelo de Toulmin, fundamentado "en una propuesta sistemática de la argumentación, pero que en la 'acomodación' colombiana las categorías como claim (tesis o proposición), data (datos que la apoyan), warrant (garantía que establece la relación válida entre datos y tesis), backing (soporte a la garantía), rebuttal (restricción al soporte), qualifier (expresión para mostrar el grado de seguridad del argumento) pueden ser modificadas, con el debido reconocimiento inicial de la ontología expresada anteriormente" (Mora, 2011).
} 
orientar el ejercicio metodológico y teórico, pero solo se encontró información relacionada con la comunicación intercultural y el bilingüismo; dada la ausencia de investigaciones y de plataformas teóricas y tecnológicas para el desarrollo de la argumentación intercultural en contextos interculturales, surgió la motivación de escribir este artículo en el cual se busca, fundamentalmente, dar una mirada crítica a los enfoques metodológicos del proyecto y aproximarnos a esta definición, con lo cual se vislumbren sus posibilidades y limitaciones en contextos multiculturales.

\section{Planteamiento de la cuestión}

Para desarrollar el problema que se plantea en este artículo, se describirá la metodología del proyecto, se analizarán enfoques teóricos de la argumentación y de la interculturalidad con el fin de tejer las posibles relaciones entre estos dos, como conceptos y como prácticas. De esta manera, el lector podrá ver que en el proyecto no solo fue importante la definición y fundamentación de la argumentación intercultural, sino que en la búsqueda teórica y en el diseño metodológico se identificaron las tensiones que generan los métodos y las lógicas occidentales en contextos educativos locales a los que pertenecen las comunidades indígenas. En este sentido, el artículo busca plantear una reflexión en torno a los modos como estamos abordando la formación en competencias argumentativas, desde las cuales se asumen mecanismos para la verificación y la demostración más cercanos a la racionalidad positivista, desconociendo otros modos de comunicación y argumentación, lo cual es característico de las estrategias comunicacionales de las comunidades indígenas. Se busca que en los sistemas educativos y con el diseño de software para argumentación haya mayor receptividad a las formas de comunicación y argumentación de las distintas comunidades. Solo podrá hablarse de la existencia de una argumentación intercultural, cuando generemos espacios de comunicación en los que los miembros de las comunidades puedan interactuar sin luchar por sobreponer o asimilar un patrón dominante.

\section{Desarrollo}

\section{Características comunicacionales e interculturalidad en la comunidad educativa}

Para comprender el escenario de esta reflexión se presentarán algunas características de los estudiantes. En la institución educativa se encuentran estudiantes de tres grupos culturales: indígenas, llaneros y colonos. Sus lugares de procedencia son, para los primeros, el Vichada; para los segundos, el Meta y para los terceros, distintas regiones del país. El trabajo se realizó con estudiantes de grado $10^{\circ}$, el cual estaba integrado por un $51 \%$ de estudiantes indígenas, $13 \%$ de estudiantes colonos y $37 \%$ de estudiantes llaneros; pese a que la mayoría de 
estudiantes son indígenas, se encontró que el español es la lengua en la que se dan los procesos de enseñanza y aprendizaje, pues se considera como la lengua en la que se produce conocimiento, como lo afirma Héctor Ramírez Cruz (2003) en su tesis de maestría: Diagnóstico sociolingüistico de Cumaribo, zona de contacto indígena, colono, Vichada: "El español es la lengua dominante, la lengua nacional, la lengua de la educación y, por lo tanto, el sistema al que todos los hablantes tienden a acceder, además de cumplir una función de "identificación", en primer lugar, con la nación y, en segundo lugar, con la comunidad latina" $(2003,31)$. No obstante, los estudiantes indígenas tienen dificultades en el manejo del español, pues muchos de ellos viven la infancia en su comunidad e ingresan al colegio en la básica secundaria, y por esta razón no se encuentran muy familiarizados ni con el ambiente escolar ni con el español, idioma en el que se transmite el conocimiento en la institución. Esta distinción se hizo evidente para nosotros en las intervenciones que los estudiantes hacían en los grupos focales y en la manera como conformaban grupos de trabajo, pues en el momento en que se les solicitó que lo hicieran, organizaron grupos solo de estudiantes sikuani o de colonos.

Las relaciones en el aula de clase se dan en términos de la multiculturalidad como coexistencia de varias culturas, o en términos de Canclini, como yuxtaposición de etnias o grupos de una ciudad o nación (2004) desde la cual se admite la diversidad de culturas pero se genera un cierto tipo de "segregación". La inquietud que surge hasta acá es sobre la suerte que corren las culturas locales con su lengua, costumbres, saberes y tradiciones frente a las culturas que se insertan dentro del sistema educativo dominante: Esperamos plantear algunas respuestas a este interrogante.

Para aclarar lo que se entiende por interculturalidad, se retoman dos planteamientos: Primero, el del investigador Axel Rojas (2005), quien considera que la interculturalidad es un proyecto político que se ha gestado en América Latina para el reconocimiento de los pueblos indígenas; y luego el de Canclini quien desarrolla una visión de la interculturalidad desde una perspectiva culturalista y la asume en dos sentidos, como estrategia metodológica y como intercambio. De ahí afirma que "interculturalidad remite a la confrontación y el entrelazamiento, a lo que sucede cuando los grupos entran en relaciones e intercambios. Ambos términos implican dos modos de producción de lo social: multiculturalidad supone aceptación de lo heterogéneo; interculturalidad implica que los diferentes son lo que son en relaciones de negociación, conflicto y préstamo recíprocos" $(2005,15)$. En este sentido, la interculturalidad tiene una connotación política que implica la lucha por la afirmación y el reconocimiento de lo propio, pero a la vez se caracteriza por el intercambio cultural. Este enfoque de la interculturalidad está condicionado por varios elementos que determinan la forma como se comunican entre sí los miembros de la comunidad, pero encontramos que el manejo de la lengua es muy importante para el desarrollo y la vivencia de la interculturalidad, asunto que se expone a continuación. 


\section{Lengua e interculturalidad}

Este ejercicio de intercambio que sugiere la interculturalidad se ve opacado cuando existe una lengua o una cultura dominante, lo que se refleja en la comunidad educativa del Colegio Sagrado Corazón de Jesús, pues allí el español es la lengua dominante, lo cual está generando un fuerte impacto en los estudiantes que provienen de comunidades indígenas, y que en este contexto provienen de una "cultura minoritaria". Este impacto se manifiesta en aspectos como la segregación y el aislamiento de los estudiantes indígenas de los demás estudiantes, y en el reconocimiento de las dificultades para expresarse en una lengua que es familiar como la propia, en este caso, el sikuani. Esta situación se observa en el planteamiento de Honorio, uno de los estudiantes Sikuani, cuando afirma: " $A$ ellos les gusta participar ¿si? En cambio a nosotros los indígenas como que no tenemos la capacidad de expresarnos bien, y pues no hablamos bien el español, entonces, no pues no tenemos esto la oportunidad de expresarnos, entonces nos comemos las palabras, o pues no podemos expresarnos bien" (2011, 29 de agosto). ${ }^{3}$

Según lo anterior, el español se presenta como lengua dominante en la institución educativa, lo que limita el empleo del sikuani en los procesos de enseñanza-aprendizaje. El español se percibe como lengua en la que se produce y se transmite la ciencia y el conocimiento, en tanto que el Sikuani es la lengua para comunicarse entre los miembros de la comunidad, para intercambiar los asuntos de interés, para expresar afectos, saberes y percepciones del mundo y para generar lazos entre ellos. El español se considera como la lengua oficial; de ahí que en el colegio debe ser aprendida porque es la lengua que maneja la mayoría de los docentes, y a través de ella se realizan los procesos de enseñanza-aprendizaje. Ramírez Cruz se cuestiona acerca de la valoración que se tiene del español por parte de los Sikuani y plantea:

¿Cómo pueden dos lenguas en contacto, que los hablantes bilingües deben seleccionar en una u otra situación, conservar una valoración positiva? En primer lugar, consideremos los valores que parecen inherentes a las lenguas: la lengua española es lengua oficial en todo el territorio y por lo tanto, con la cual puede accederse, por lo menos socialmente, al contexto nacional, es la lengua de la educación, por lo tanto la lengua del conocimiento, de las ciencias, de las artes; la lengua sikuani no parece, en primera instancia tener más valor que el que desde su subjetividad le otorguen los hablantes: es la lengua del grupo, es la lengua que se aprende en casa. Tiene además una valoración que consideraremos, por ahora, impuesta, y que puede influir en la valoración positiva que de ella se hace: es lengua oficial en el territorio y es asignatura obligatoria en los colegios porque La Constitución Nacional así lo establece $(2003,91)$. 
En este caso se distinguen usos de la lengua que responden a necesidades específicas; por eso el sikuani se percibe y se utiliza como la lengua a través de la cual se transmiten afectos y modos de comprensión muy particulares de la comunidad. En términos de Ramírez Cruz, "el español cumple mayoritariamente con todas las funciones del lenguaje (más aún en los niños), y el sikuani queda rezagado, casi en forma exclusiva, a la función poética y, levemente, a la directiva" (2003, 83). Por eso para los estudiantes del colegio es fundamental el aprendizaje del español en la medida en que a través de este se comunican y se produce el conocimiento. Sin embargo, se presentan dificultades para el manejo de una sola lengua y por este motivo se conforman grupos aislados en el aula de clase, grupos constituidos por sikuani, y otros, solo por colonos. En palabras de María, los sikuani no participan activamente porque:

Les da pena, de pronto, que nosotros de pronto nos empecemos a burlarnos de cómo ellos van a opinar sobre el tema o las ideas que van a plantear ellos, porque a veces somos muy burlones, es la verdad, (...) y entonces tal vez como ellos hablan o como se expresan o de pronto por las ideas que dan, pero esa es la falta de tono, o sea ¿sí? Deberían de dejar a un lado la pena para poder crecer más adelante ¿no?, para progresar (2011, 29 de agosto). ${ }^{4}$

Para Ramírez Cruz (2003, 83), en estos casos se presenta una situación de diglosia en la medida en que cada lengua tiene una función determinada. Por ejemplo, el español se utiliza para las situaciones de mayor formalidad, distantes a la comunidad, mientras que el sikuani se emplea para las circunstancias de mayor proximidad, de carácter informal e íntimo. Debido a esto y al riesgo de que los estudiantes dejen de utilizar el sikuani poco a poco, las directivas del colegio empezaron a implementar desde 2010 la cátedra de Sikuani. Hoy todos los estudiantes han aprendido a cantar el Himno Nacional de Colombia en español y en Sikuani, como una forma de incorporar la lengua en los procesos educativos y empezar así a preparar el terreno para el bilingüismo y la interculturalidad.

En esta situación de diglosia, en la que cada lengua tiene una función específica, ¿cómo se puede dar una argumentación intercultural? Podríamos decir que esta sería prácticamente imposible en la medida en que se debe argumentar con el código de la lengua dominante, y que en este sentido el estudiante debe aprenderla y olvidar la propia. Desde una perspectiva intercultural, siguiendo lo que afirma Canclini, la interculturalidad se debe dar en términos de relaciones de negociación, conflicto y préstamo recíprocos entre culturas heterogéneas. Ello incentiva la afirmación de cada sujeto en lo propio, pero en la apertura a lo ajeno, sin necesidad de que exista una cultura que limite o que absorba a la otra, lo que sucede en un contexto educativo monolingüe. 


\section{Del manejo de la lengua a una argumentación intercultural}

"Si queremos descolonizar la colonialidad del poder/saber y no ser subsumidos y silenciados, las formas disciplinarias mismas, sus metodologías y tecnologías de producir y representar los discursos tienen que ser descolonizadas."

Catherine Walsh, Freya Schiwy y Santiago Castro-Gómez (2002).

Cuando en este proyecto se planteó la argumentación intercultural se tenía como punto de partida una concepción occidentalizada de la comunicación y de la producción de conocimiento. La cultura occidental se ha expandido mediante el uso de estrategias para hacer cambiar de actitud al otro, para persuadirlo, convencerlo y hasta a veces obligarlo a realizar ciertas acciones. Desde el proyecto, lo que se pensó inicialmente como argumentación intercultural no se ha dado en la historia de occidente, pues lo que se encuentra son ejemplos de cómo se ha tratado de incidir y persuadir para lograr cambiar la conducta del otro mediante la aplicación de estrategias de dominación y subordinación, asunto que se ha estudiado muy bien desde el proyecto modernidad/ colonialismo. Por lo tanto, no se han dado escenarios de debate o de negociación sino de uso de estrategias para persuadir, apelar a las emociones o a la fuerza y lograr así el cambio en la forma de vida, en el comportamiento o en el pensamiento del otro.

El propósito de la argumentación incluye diferentes enfoques. Según Perellman y Olbrechts- Tyteca, la finalidad de la argumentación es "provocar o acrecentar la adhesión de un auditorio a las tesis que se presentan a su asentimiento" (1958, 7). Para Álvaro Díaz $(2002,6)$, con la argumentación se busca convencer, modificar alguna opinión del auditorio, mientras que Habermas (2010) la asume de modo negativo y por ello plantea que en la situación ideal de argumentación los participantes están en condiciones iguales de poder, de modo que no existan actitudes manipulativas o estratégicas para convencer, incidir o cambiar la actitud del otro. Para ello, distingue entre la acción comunicativa y la acción estratégica, pues la primera busca llegar al mejor juicio, que tiene una aceptación intersubjetiva, y la segunda plantea la argumentación como modo de incidir en el pensamiento o en la conducta del otro. Por esta razón, para precisar el enfoque del proyecto, la visión de la argumentación que más se relaciona con lo que acá asumimos como interculturalidad es la habermasiana, ya que permite conservar su carácter político e ideológico en escenarios de discusión y debate en los que no existen relaciones jerárquicas. Para ello se requiere que todos los miembros de la comunidad sean bilingües y además, conscientes del significado de habitar en un contexto intercultural que debe generar relaciones de intercambio basadas en principios éticos.

Según esta relación teórica, la argumentación intercultural es posible en escenarios en los que se permita el debate, la discusión, la divergencia y la exposición de 
puntos de vista diferentes, de distintos modos de entender la realidad. Se trata, en suma, de construir una argumentación de carácter dialógico que permita la interacción de personas con puntos de vista diferentes, y para ello el software Dígalo no es una herramienta muy útil en la medida en que el tipo de argumentación que sugiere es de carácter monológico, porque es en el ejercicio dialógico (Herrero, A., 2001) donde se pueden encontrar diferentes puntos de vista, y así llegar a acuerdos o a negociaciones, condiciones para que se pueda dar una verdadera argumentación intercultural.

Con lo anterior no se propone contradecir las ventajas del software Dígalo y del desarrollo de la argumentación en los contextos escolares, pues a partir de allí se puede lograr el reconocimiento y la interacción de las comunidades, las costumbres, los saberes y la lengua propia, elementos que pueden mejorar los procesos de interculturalidad. No obstante, en este artículo se busca cuestionar el punto de referencia epistemológico que fundamenta este trabajo de investigación y su diseño metodológico, ya que legitima todo un proyecto de dominación desde occidente. Desde el proyecto modernidad/colonialismo y desde la perspectiva de las ciencias sociales se puede decir que:

La carga moderna/colonial de las ciencias sociales y humanas, sus teleologías inmanentes proponen un solo camino de desarrollo que sigue el modelo europeo y ubican a los proyectos alternativos en escalas temporales de atraso frente a la modernidad. Al mismo tiempo se establece la racionalidad, superioridad y normalidad de ciertos grupos y conocimientos y la subalternidad de otros (Walsh, C. et. al., 2002, 13).

En la metodología del proyecto se vio cómo una lengua subordina a la otra, una cultura subordina a la otra, y termina reproduciendo un modelo hegemónico de pensar, educar y producir conocimiento, anulando lo propio de las comunidades, que fueron las metodologías empleadas en nuestro caso. Por esta razón, los autores proponen indisciplinar las ciencias sociales pues: "implica el reconocimiento de otras formas de conocimiento, particularmente los conocimientos locales producidos desde la diferencia colonial, y los entrecruces y flujos dialógicos que pueden ocurrir entre ellos y los conocimientos disciplinares" (Walsh, C. et. al., 2002, 13).

En el caso que nos ocupa, el problema que se evidencia no es la necesidad de argumentar para convencer y superar otras visiones del mundo, sino de establecer mecanismos y escenarios para la discusión de temas de interés colectivo que permitan la comunicación en lenguajes verbales y no verbales y la construcción de símbolos y códigos que respondan a un proyecto educativo y político que hagan de la riqueza cultural y de conocimiento, una de las fortalezas de la región, lo que no se logra mediante las didácticas que se utilizan en la escuela tradicional. 


\section{Visiones criticas}

Como se dijo al comienzo de este artículo, se busca realizar un ejercicio crítico que nos permita como investigadores, evaluar los métodos que utilizamos para el trabajo con diferentes comunidades, pues desde una mirada positivista, todos los métodos servirían para distintas situaciones. Esto nos llevaría a desconocer la naturaleza de los contextos y a generar soluciones homogéneas desde las cuales se termine anulando la diferencia cultural.

Por ello, desde esta perspectiva crítica y autocrítica, nuestro proyecto de investigación tampoco se encontraba diseñado desde el punto de vista intercultural, ya que cuando propusimos el desarrollo de una argumentación intercultural en un grupo de estudiantes del colegio, estábamos legitimando, reforzando y dando continuidad al uso del español para situaciones de debate y discusión, en primer lugar, porque el software está adaptado al español pero no al sikuani y en segundo lugar, porque se desconocían las características comunicacionales de los miembros de esta comunidad.

Otro elemento crítico en este ejercicio, consistía en que los investigadores no manejaban el sikuani, y por ello la argumentación intercultural se planteó desde el español como la lengua del conocimiento, del aprendizaje, como lengua que conlleva una lógica hegemónica. Por ello, en el enfoque metodológico del proyecto era necesario establecer estrategias que permitieran la coexistencia de las perspectivas culturales existentes, y no que hubiera una lógica que absorbiera a la otra, como finalmente sucedió. Así, encontramos que en el diseño metodológico del proyecto había fundamentaciones que de antemano impedían el desarrollo de la argumentación intercultural porque estaba concebido desde el enfoque positivista de los métodos de investigación social aplicados a la educación. Así se evidenció en el primer ejercicio realizado con la comunidad, en el que se desarrolló la dinámica de Espina de Pescado con el fin de construir los casos desde los intereses de la comunidad. El problema de esta técnica es que está basada en las relaciones de causa-efecto, que condiciona el modo de pensar de los estudiantes y hace que se visualice una sola forma de entender los contextos. En estas condiciones, se terminó afirmando el punto en el que estaban ubicados los investigadores, pero no los casos de mayor prioridad para los estudiantes. Después se realizó un grupo focal con el fin de que ellos se familiarizaran con el ejercicio de discusión y de argumentación, y posteriormente se buscó la discusión a través del uso del software Dígalo.

Como se dijo antes, una de las metas del proyecto era la identificación del modo como sería posible una argumentación intercultural a través del software Dígalo, pero uno de los mayores obstáculos fue la infraestructura tecnológica, pues en el momento de realizar el ejercicio, la sala de sistemas no se encontraba habilitada para trabajar en red. Por esta razón se realizó la actividad con el software pero con un solo computador para todos los estudiantes, lo que no nos permitió alcanzar 
este objetivo del proyecto. Lo anterior significa que el diseño metodológico del proyecto se realizó por fuera del contexto previsto. Los investigadores planeaban desde sus escritorios en la ciudad de Bogotá, y posteriormente iban a Cumaribo a aplicar las técnicas programadas en la ciudad. Por ello, el tema de la argumentación intercultural no se exploró a profundidad, máxime cuando se pretendía hacerlo a través del uso de un software y cuando las brechas digitales impedían su instalación y uso por parte de cada estudiante. ${ }^{5}$

Así pues, se han expuesto aspectos críticos en relación con el diseño metodológico del proyecto, desde los cuales se presentaba con dificultad el desarrollo de lo que denominamos argumentación intercultural. Ahora se presentarán algunos de los aportes y conclusiones a partir de esta reflexión.

\section{Conclusiones}

Con este proyecto se observó cómo en la escuela se pone en juego una serie de relaciones que están por fuera de la planeación académica y que caracterizan los aspectos culturales de los actores de una comunidad, lo cual debería afectar los planes de estudio mediante la generación de currículos flexibles y didácticas que permitan las necesidades particulares de los grupos culturales. No obstante, el sistema educativo colombiano está diseñado a partir de estándares generalizadores y homogeneizantes cuya aplicación desconoce las particularidades de los diferentes contextos escolares. Por ello, en pocos casos hay diferencia en las estrategias para la enseñanza y el aprendizaje y en las competencias que se busca desarrollar, como es el caso de las habilidades para la ciencia y las "competencias comunicativas”, dentro de las cuales está la argumentación. De igual modo, no encontramos particularidades en la implementación de software, pues para ello se requeriría un equipo interdisciplinario que primero identificara las características comunicativas de las comunidades y posteriormente desarrollara el software según estas características.

Así mismo encontramos estandarización en los métodos que utilizamos para abordar la investigación en estos contextos, porque, como investigadores, muchas veces tomamos las técnicas de recolección de información que ya están establecidas y las aplicamos sin cuestionar si tienen relación con los contextos, con sus propias lógicas y con las formas de actuación de las diferentes comunidades. El verdadero valor de una investigación radica en buscar el impacto en la comunidad, en comprender la naturaleza del contexto y 
en diseñar las estrategias que se relacionen con los modos de ver el mundo de la comunidad para que terminen apropiándose de ellas.

En términos del proyecto modernidad/colonialismo, se hace necesario buscar las fuentes de la dominación y desde allí establecer otras metodologías, didácticas y horizontes teóricos que nos permitan descolonizarnos, para contrarrestar la reproducción del sistema educativo homogeneizante, que solo acepta la multiculturalidad para integrarla y volverla una sola, aún con el riesgo de perder la diferencia que caracteriza una cultura. En este sentido, para este tipo de investigaciones es fundamental incorporar metodologías como la Investigación, la Acción, la Participación, la teoría fundamentada o las utilizadas en los estudios culturales, pues con ellas se permite escuchar, visualizar y confrontar las características de los contextos.

El uso de Dígalo puede resultar útil, siempre y cuando existan varios aspectos: 1) infraestructura tecnológica para su funcionamiento, 2) un software que se adapte a las condiciones comunicativas de la comunidad, que se pueda escribir en sikuani y en español, y 3) una comunidad que se familiarice previamente con la herramienta y que se comunique de manera oral y escrita en ambas lenguas. En la actualidad, el software está diseñado para modificar algunas de las estructuras de comunicación, pero no para la escritura en esta lengua, y por ello en su uso tendrá que utilizarse el español o el inglés.

La argumentación intercultural puede llegar a ser una propuesta de carácter comunicativo y ético que permita la relación y el intercambio entre diferentes culturas. Para poder lograrlo se requieren transformaciones sustanciales en el sistema educativo, desde el currículo, la didáctica, la forma de evaluación y las metodologías de investigación que permitan visibilizar las relaciones interculturales en términos de su complejidad y del alcance en la toma de decisiones en un contexto sociocultural.

Actualmente, la ventaja que se encuentra es que existen políticas para el reconocimiento de los saberes y para la protección de las lenguas y de los grupos indígenas. Aún así, el camino por recorrer para lograr una argumentación intercultural es largo. Implicaría inicialmente, que las comunidades educativas se apropiaran de la perspectiva intercultural y que a partir de ella diseñaran sus propios currículos, determinaran los contenidos y las estrategias de evaluación y que hubiera una articulación de las políticas de evaluación nacional. Por ejemplo, las pruebas Icfes actualmente no se ajustan a las necesidades de las comunidades sino al manejo de un conocimiento general y homogéneo para todos los estudiantes del país, cuyos resultados evidencian grandes desniveles generados por las cosmovisiones particulares y por las características de los contextos. 


\section{Referencias bibliográficas}

Camps, A. (1995). Aprender a escribir textos argumentativos: Características dialógicas de la argumentación escrita. Revista de Comunicación, Lenguaje y Educación, 25, 51-63.

Díaz, Á. (2002). La argumentación escrita. Medellín: Editorial Universidad de Antioquia. Gómez, H. (2011). Grupo focal integrado por Vargas, A. \& Méndez, C. Manuscrito no publicado, Cumaribo (Vichada).

González, J. H. Discernimiento: Evolución del pensamiento crítico en la educación superior. El proyecto de la universidad ICESI, [en línea]. Disponible en: http://www. eduteka.org/pdfdir/DiscernimientoHipolitolcesiCapitulo1.pdf [2011, 11 de marzo].

Habermas, J. (2010). Teoría de la acción comunicativa. Madrid: Trotta.

Perelman, C. (2006). Tratado de la Argumentación: La Nueva Retórica. Madrid: Gredos.

Mora, P. (2011). La herramienta "Digalo", fuente de argumentación en el aula, [ en línea]. Disponible en: http://www.virtualeducaperu.org/index.php?option=com_ content\&view=article\&id=78:Ia-herramienta-qdigaloq-fuente-de-desarrolloargumental- en-el-aula\&catid=38:articulosboletin\&ltemid=76 [2011, 10de mayo].

Ramírez Cruz, H. (2003). Diagnóstico sociolingüístico de Cumaribo, zona de contacto indígena - colono, Vichada. Bogotá: Universidad Nacional de Colombia. Facultad de Ciencias Humanas.

Rojas, A. (2005). Inclusión social, interculturalidad y educación. ¿Una relación (im) posible?, [en línea]. Disponible en: http://www.foro-latino.org/flape/foros_virtuales/ doc_fv_4/ FV4-Conclusiones.pdf [2010, 3 de abril].

Vargas-Mendoza, J. E. (2007). La Argumentación Inductiva de Toulmin. Ciudad de México: Asociación Oaxaqueña de Psicología A .C., [en línea]. Disponible en: http:// www. conductitlan.net/toulmin.ppt [2011, 1 de marzo].

Walsh, C., Schiwy, F.y Castro-Gómez, S. (Editores), (2002). Indisciplinar las ciencias sociales. Geopolíticas del conocimiento y colonialidad del poder. Perspectivas desde lo andino. Quito: Universidad Andina Simón Bolívar, Abya-Ya. 